\title{
Amplification through losses in nonlinear fiber optics
}

\author{
Auro M. Perego ${ }^{1, *}$, Sergei K. Turitsyn ${ }^{1,2}$ and Kestutis Staliunas ${ }^{3,4}$ \\ ${ }^{1}$ Aston Institute of Photonic Technologies, Aston University, Birmingham, B4 7ET, UK \\ ${ }^{2}$ Novosibirsk State University, Novosibirsk 630090, Russia \\ ${ }^{3}$ Institució Catalana de Recerca i Estudis Avançats, Pg. Lluis Companys 23, 08010, Barcelona, Spain \\ ${ }^{4}$ Departament de Física i Enginyeria Nuclear, Universitat Politècnica de Catalunya, Rambla Sant Nebridi 22, 08222 \\ Terrassa, Barcelona, Spain
}

\begin{abstract}
We present the most recent results about a novel modulation instability of a continuous light wave in a normal dispersion nonlinear optical fiber, induced by spectrally asymmetric losses for signal and idler waves. The presence of such spectrally asymmetric losses profile causes energy transfer from the pump to both signal and idler. A variety of applications concerning both amplification of signals and generation of pulses is discussed.
\end{abstract}

\section{Keywords-modulation instability, optical amplification.}

Modulation instabilities, are ubiquitous processes in nature and in technological devices where they are linked to the spontaneous amplification of certain spectral modes whose amplitude grow exponentially eventually leading to a modulation of the homogeneous state of the system. A variety of instability mechanisms have been individuated in the study of nonlinear science in general and in nonlinear fiber optics in particular. Benjamin-Feir instability for instance is the basic operational principle behind the fiber optics parametric amplifier [1], while Faraday instabilities, induced by parametric modulation in dispersion oscillating fibers suggest the possibility of an interesting frequency selective amplification [2].

a)

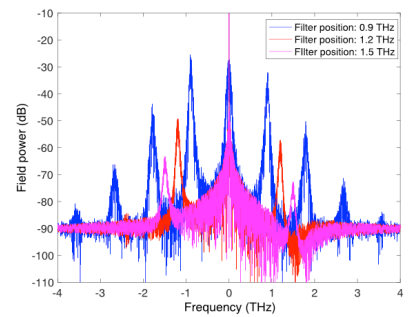

Both Benjamin-Feir and Faraday modulation instabilities occur when certain phase-matching or quasi-phase-matching conditions are satisfied. In fibers where the group velocity dispersion is normal and not modulated, the continuous wave solution is stable. We present here the counterintuitive and striking phenomenon of gain-through-losses: if dissipation is applied asymmetrically for signal and idler waves, for instance if losses are applied on the signal frequency only, this leads surprisingly to the amplification of both signal and idler waves. Increasing the dissipation in a certain spectral region favours energy transfer from the pump frequency to that lossy region [3]. Such powerful principle can be implemented both through distributed and lumped filters and can be used to create tunable amplifiers, allowing a mapping of the dissipation profile into a gain profile (imaging of losses into gain). Potential applications in ring fiber resonators for the generation of frequency combs and pulses are discussed too. Furthermore the gain-throughlosses process can be exploited in optical parametric oscillators to achieve amplification when it is not possible to satisfy standard phase-matching conditions, and it is expected to be a universal dynamics of nonlinear systems even outside photonics.

b)

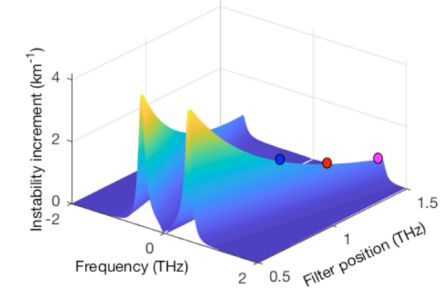

Fig. 1. In a) the recorded spectrum is plotted after a $4 \mathrm{~km}$ numerically simulated propagation of the electric field in a single mode fiber with nonlinearity coefficient , $\gamma=15(\mathrm{~W} \mathrm{~km})^{-1}$ and group velocity dispersion $\beta_{2}=1 \mathrm{ps}^{2} \mathrm{~km}^{-1}$ in presence of a distributed filter damping modes around frequency 0.9 (blue curve), 1.2 (red curve) and 1.5 (pink curve) $\mathrm{THz}$ respectively. Initial condition at the beginning of the fiber is continuous wave radiation with power equal to $5 \mathrm{~W}$. The filter induces sidebands amplifications for damped modes (signal) and symmetric ones with respect to the pump (idler); higher harmonics are amplified too. In b) the theoretical predictions for the instability growth rate are plotted showing, in agreement with numerical simulations, that the process is more efficient the closer the filter position is to the pump frequency (instability is predicted when the increment is larger than zero). Dots on the plot correspond to cases considered in a).

\section{REFERENCES}

[1] M. Mahric, Fiber optics parametric amplifiers and related devices. Cambridge Univesity Press: Cambridge, 2007.
[2] A. Mussot, M. Conforti, S. Trillo, F. Copie, and A. Kudlinski, "Modulation instability in dispersion oscillating fibers", Advances in Optics and Photonics, vol. 10, pp. 1-42, 2018.

[3] A. M. Perego, S. K. Turitsyn and K. Staliunas, "Instabilities and gain through losses in nonlinear optics", Submitted for publication, 2018. 